\title{
INVESTIGACIÓN
}

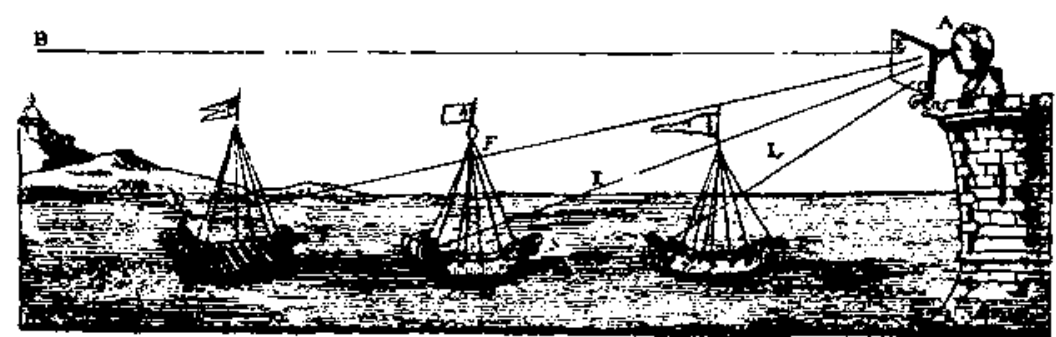

$\mathbf{Y}$

EXPERIENCIAS

DIDÁCTICAS

\section{REFLEXIONES EN TORNO A LA LÍNEA EDITORIAL DE LA REVISTA ENSEÑANZA DE LAS CIENCIAS}

SANMARTí, N. ${ }^{1}$ y AZCÁRATE, $C .{ }^{2}$

' Directora de Enseñanza de las Ciencias.

2 Directora adjunta de Enseñanza de las Ciencias.

\section{SUMMARY}

The aim of this article is to inform about some thoughts and decisions taken by Enseñanza de las Ciencias journal editorial staff about the criteria for selecting articles. We believe that this criteria should be discussed in order to make possible an effective response to the needs of the teaching and research communities that constitute the public of the journal.

\section{INTRODUCCIÓN}

A través de sus años đe existencia, la revista Enseñanza de las Ciencias se ha consolidado como un punto de referencia importante en los países de habla hispana para la comunicación de la investigación en el campo de la didáctica de las ciencias experimentales y de las matemáticas. 
Desde el primer número de la revista aparecido en 1983 , las universidades españolas han experimentado grandes cambios. Entre ellos se puede destacar la creación de los departamentos de didáctica de las ciencias experimentales y de las matemáticas, con la consiguiente aparición de programas de doctorado en dichas áreas que han supuesto un importante impulso a la investigacion.

En estos dos últimos años el consejo de ređacción đe la revista frecuentemente se ha planteado la pregunta: ¿Qué hacer ante la gran cantidad de artículos que se reciben, muchos de los cuales son el resultado de prácticas o de reflexiones sin duda interesantes para las personas que los redactan, pero no siempre para los que las han de leer o para el desarrollo de la didáctica de las ciencias?

Cada investigación, cada reflexión, responde a un mundo particular importante para los que la han llevado a cabo. El consejo editorial de cualquier revista selecciona los que cree más interesantes para el futuro de su campo de investigación, por lo que, de alguna manera, se enfrenta a un compromiso ético. Al aceptar unos artículos y denegar otros está condicionando: determinadas líneas de investigación, la propia política educativa de un país en enseñanza de las ciencias, la forma de trabajar en las aulas y, -tampoco hay que olvidarlo-, qué profesores se seleccionan en la universidad para enseñar didáctica de las ciencias, o a cuáles de cllos se mejora su estatus, ya que se valora la publicación de artículos en determinadas revistas como indicativo de calidad.

En los últimos años, diversas revistas dedicadas a la publicación de artículos de investigación en didáctica de las ciencias o de las matemáticas se han planteado similares reflexiones, como, por ejemplo, el Journal of Research in Science Teaching (Smith, Wandersce y Cummins, 1993), el Educational Studies in Mathematics (Dörfler, 1993), el Journal for Research in Mathematics Education (Lester, 1993), el Science Education (Duschl, 1994), el International Journal of Science Education (Gilbert, 1994), el Educational Research (Donmoyer, 1996), etc.

Por todo ello, nos ha parecido necesario efectuar nuestra propia reflexión y comunicarla a los lectores.

\section{PREGUNTAS Y RESPUESTAS}

Cualquier retlexión sobre la línea editorial de una revista se enfrenta a la necesidad de tomar posiciones ante los numerosos dilemas que se presentan en el momento de orientar una determinada línea editorial.

Algunas de las preguntas que nos hemos planteado son:

- ¿A quién se dirige la revista: al profesorado de aula o al profesorado investigador universitario?

- ¿Qué tipo de artículos se deberían publicar?
- ¿Qué referentes teóricos? ¿Un sólo parađigma o varios?

- ¿Qué temáticas?

- ¿Qué metodología? ¿Cómo estructurar los artículos?

\section{A quién se dirige la revista: al profesorado de aula o al profesorado investigador universitario?}

La mayoría de los consejos de redacción de las revistas de investigación en el campo de la enseñanza han de plantearse resueltamente si una publicación de tales características puede ser útil al mismo tiempo a la comunidad de investigadores, que quiere comunicar los resultados de sus trabajos y conocer los de otros, y a la comunidad de enseñantes, que desea mejorar su práctica y abrir su campo de reflexión.

Nadie duda de la utilidad de una revista de investigación en didáctica de las ciencias y de Ias matemáticas como vehículo de comunicación entre los investigadores de estos campos de conocimiento. Pero, al mismo tiempo, Enseñanza de las Ciencias nació con la finalidad de ser leída mayoritariamente por el profesorado de los centros educativos no universitarios y, consecuentemente, con el objetivo de que su contenido permiticra mejorar la práctica docente.

Es bien conocido que, en general, la investigación educativa se relaciona muy poco con dicha práctica (Gimeno Sacristán, 1983). Sus resultados tardan muchos años en ser conocidos por el profesorado, y pocas veces son aplicados de forma generalizada. Ésta es una de Ias causas que llevaron al desarrollo de los modelos de la Ilamada «investigación-acción», pero sobre este tipo de investigaciones se escriben pocos artículos y no siempre tienen un interés generalizado.

El profesorado acostumbra a buscar en una revista aquella idea que conecte con sus puntos de vista y con sus necesidades, y que le sea útil tanto para entender $\mathrm{el}$ sentido de muchas de las «intuiciones» que ya ha aplicado en sus clases como para sugerirle otras. Filo comporta que la relación teoría-práctica en el campo educativo está condicionada por dos aspectos muy importantes:

- Primero, por la necesidad de coincidir en el modelo didáctico teórico de referencia. Toda investigación tiene unos referentes que, si no son compartidos, es imposible llegar a reconocer su aplicabilidad. Un descubrimiento importante en didáctica de la ciencias o de las matemáticas conlleva no sólo un cambio en la forma de actuar, sino también en la forma de pensar. Y se sabe lo difícit que es cambiar las propias concepciones. Por ello no ha de extrañar que los resultados de la investigación didáctica tarden tantos años en generalizarse.

- Segundo, por el hecho de que el contexto de la práctica educativa está cambiando continuamente. Cada clase, cada alumno, cada escuela, cada sociedad es distinta. No hay recetas para enseñar a sumar o para enseñar el cambio químico, ya que lo que es útil para Juan no lo es 
para Ana, y aquello que «funcionó» en la clase A no sirvió en la clase B. Por tanto, los restitados de una investigación no son útiles porque se puedan aplicar directamente, sino porque presentan marcos de referencia de los que se pueden deducir aplicaciones en diferen. tes contextos.

Consecuentemente no resulta fácil reconocer la «aplicabilidad» de una determinada investigación educativa. A ello se debe añadir que si bien es evidente que la didáctica de las ciencias o de las matemáticas tiene por objetivo último mejorar la práctica educativa, los inves. tigadores buscan además en una revista la oportunidad de dar a conocer sus trabajos y sus puntos de vista, así como el de conocer el de otros compañeros y compañeras. Se puede afirmar que se escribe más pensando en la comunidad de investigadores y no tanto en la del profesorado en activo.

Por todo ello, creemos que es necesario promover que los artículos publicados, fundamentalmente:

a) planteen problemas que conecten con Ias preguntas, dudas y necesidades del profesorado que día a día ha de enfrentarse al reto de enseñar ciencias y matemáticas significativamente;

b) tengan un marco teórico definido, argumentado y coherente con la reflexión o investigación analizada;

c) sugieran o propongan alternativas a formas de pensar o actuar fundamentadas en relación con el marco teórico o con los datos empíricos.

\section{2. ¿Qué tipo de artículos?}

En el consejo de redacción de la revista se reciben artículos de todo tipo. Muchos de ellos tratan de experiencias de clase no analizadas en profundidad, de proyectos de programas de asignaturas, de un tema o de actividades de aula no experimentadas, de reflexiones teóricas sin datos empíricos que las avalen, de análisis estadísticos de respuestas de alumnos o de profesores sin un marco teórico de referencia consistente, etc.

Este tipo de trabajos, $\iota$ se pueden considerar investigaciones?, ¿son artículos para ser publicados en la revista Enseñanza de las Ciencias? Éstas son preguntas que también conviene responder.

Tal como indica Dörfler (1993), es difícil establecer una línea de separación clara entre una investigación y otro tipo de trabajos, a pesar de que el sentido común da un significado a este término: Una investigación es un tipo de análisis y de organización sistemática y reflexionada de las experiencias humanas, de su descripción y estructuración, de su explicación teórica y predicciones viables. Generalmente también se considera que una investigación ha de aportar alguna idea original.

Kilpatric, ya en 1981, al analizar la investigación en el campo de la enseñanza de las matemáticas, distingue entre los estudios que se caracterizan por su rigor o por su significatividad. Los primeros, dice, están diseñados para cumplir un propósito bien definido y por la utilización de técnicas que otros pueden reproducir y así validar los resultados obtenidos. Los segundos son aquellos que pueden tener un impacto sobre el pensamiento y que abren nuevas perspectivas. En ellos las preguntas formuladas son importantes y los resultados posibilitan el planteamiento de nuevas ideas y problemas.

En las discusiones metodologicas de la última "Summerschool in Science Education» celebrada en Barcelona (septiembre de 1996), se analizaron algunas de las características de lo que se podría considerar una buena investigación como, por ejemplo, el hecho de que «explica» más que describe. El explicar para que algo pueda ser comprendido implica crear diferencias y crear lenguaje, demostrar y, de alguna manera, transformar y desarrollar el conocimiento.

En Enseñanza de las Ciencias creemos que caben todo tipo de artículos que tengan estas características. Una investigación didáctica es más que describir categorias, comparar grupos o responder a preguntas cuya respuesta ya se conoce antes de empezar la investigación. Requiere explicar los datos obtenidos, Ios razonamientos emitidos o las propuestas planteadas, y abrir nuevos caminos para la reflexión y para la práctica.

Por ello serắn de interés aquellos artículos que:

a) recojan investigaciones en las que se creen explicaciones teóricas sustentadas en datos experimentales;

b) analicen experiencias de aula surgidas a partir de determinados referentes teóricos;

c) propongan alternativas a formas de trabajo o de pensamiento fundamentadas en referentes teóricos y/o experimentales;

d) revisen el estado de la cuestión en subcampos de investigación en didáctica de las ciencias o de las matemáticas.

\section{3. ¿Qué referentes teóricos? ¿Un sólo paradigma o varios?}

Es conocido que cada revista de investigación tiende a postular más o menos explícitamente determinados planteamientos teóricos y metodológicos. Y que los artículos que se publican tienden a reproducir los mismos argumentos en sus justificaciones. Por ejemplo, en Enseñanza de las Ciencias, un buen numero de artículos incluye expresiones como «según el paradigma constructivista» o ues necesario promover el cambio conceptual y metodológico», las cuales son utilizadas como principal vía argumental tanto del diseño de la investigación como de las conciusiones presentadas.

Éste es un hecho razonable e incluso necesario, ya que las personas que quieren publicar en ella o que la leen 
tienen unos referentes que le permiten inferir y compartir muchas de las aportaciones. Y no hay duda de que, en cada momento histórico, existen unos marcos teóricos más compartidos que otros por la comunidad de investigadores y, consecuentemente, la adhesión a ellos facilita la publicación de artículos.

Aun así, en campos de conocimiento como los de la didáctica de las ciencias y de las matemáticas, que están en un proceso evolutivo muy acelerado, es necesario que cualquier revista esté abierta a modelos e interpretaciones diversas e incluso contradictorias. Las ideas, los argumentos, quedan rápidamente desfasados y es necesario matizarlos, reformularlos o incluso cambiarlos. Así, actualmente, hablar de paradigma constructivista no es ningún referente ya que hay muchas formas distintas de modelizarlo.

Al mismo tiempo, no hay cuda de que uno de los problemas de cualquier artículo es que, cuando se publica, se refiere a trabajos a menudo iniciados bastantes años antes. Por ejemplo, una tesis doctoral puede durar más de cuatro años y publicar un artículo en relación con sus resultados puede necesitar dos o tres años más. Es decir, entre el momento del inicio del planteamiento del problema y la publicación del artículo escrito sobre la investigación realizada, pueden transcurrir siete u ocho años, lo que a veces comporta que el problema analizado y su marco teórico, muy significativo cuando se plante6, ya no tenga tanto interés en el momento de su publicación.

Para paliar estos condicionamientos creemos que la revista Enseñanza de las Ciencias debe potenciar los debates en torno a los temas de discusión de la comunidad en un determinado momento y en el que puedan ser planteados diferentes puntos de vista. Por ello, se incluirá un apartado con tal objetivo, en el que se potenciará la revision de las distintas formas de abordar el problema objeto de debate.

\section{4. ¿Qué temáticas?}

Mayoritariamente, Los artículos recibidos se refieren a temas relacionados con la enseñanza de la física $(50 \%)$ $\mathrm{y}$, en menor número, de la química. Muy pocos son del campo de la enseñanza de la biología y de matemáticas y aun menos de la geología o de temas transversales o interdisciplinares. Creemos que esta distribución debiera tenderse a igualar.

De la misma forma, casi todos los artículos se refieren a Ia enseñanza de las ciencias o de las matemáticas en secundaria o primer curso de universidad. Actualmente se observa un fuerte incremento de trabajos referidos a concepciones epistemológicas y didácticas de estudiantes de magisterio. En cambio, no se abordan trabajos relacionados con la escuela primaria ni con cursos avanzados de estudios científicos universitarios.

La mayor proporción de artículos recibidos aún se refieren más a la descripción de ideas de estudiantes de secundaria y de magisterio que a las formas de trabajar en función de dichas ideas. Seguramente, como indica Duschl (1994), es tiempo de admitir que algunas temáticas, problemas o preguntas deben ser abandonadas. Creemos que, después de un largo periodo de desarrollo de investigaciones básicamente descriptivas, interesa promover trabajos interpretativos que permitan avanzar en la comprensión de problemas significativos relacionados con el aprendizaje científico.

Ya en el editorial de la revista de noviembre de 1994 , señalábamos que «enseñar es una compleja experiencia en la que tiene importancia conocer qué piensan los alumnos o profesores, pero también, qué se hace en el aula, cómo los enseñantes explican y escriben y qué se comprende, como los experimentos y la resolución de problemas favorecen el aprendizaje, cómo se organizan los alumnos e interaccionan, de qué se habla en el aula, cómo influyen los aprendizajes realizados fuera de ella, qué criterios se utilizan para seleccionar y organizar las actividades y los contenidos, etc.

"Interesa promover estudios que respondan a las necesidades del profesorado de ciencias y matemáticas y profundicen en el impacto de diferentes prácticas educativas ya sea en el aula o en contextos informales. Hay pocos estudios, relacionados con la enseñanza y aprendizaje de contenidos científicos y matemáticos, que analicen la gestion y las interacciones en el aula -trabajo en pequeño o gran grupo, cooperación y trabajo individual, discurso del enseñante y del alumnado, etc.-, el grado de implicación del estudiante en el aprendizaje, su autonomía o dependencia, Ia atención a la diversidad de intereses y niveles de los estudiantes de un grupo-clase, el diseño y aplicación de actividades de diferentes tipos, la regulación de los errores en el proceso de aprendizaje, etc.»

Insistimos en que son propuestas que continúan vigentes.

\section{5. ¿Qué metodología? ¿Cómo estructurar Ios artículos?}

Muchas veces se piensa que la calidad de una investigación es valorada fundamentalmente en función de los métodos de recogida y de análisis de los datos utilizados. Pero ya se ha indicado que, aunque el rigor es una variable importante para valorar la calidad de un escrito, si al mismo tiempo no responde a una pregunta o problema significativo, dicha condicion no es suficiente.

La calidad de un estudio no debe asociarse necesariamente a estudios cuantitativos. Muchos de estos trabajos conducen a fragmentar artificialmente la realidad, a tener en cuenta una o pocas variables o a plantear hipótesis poco relevantes. Pero tampoco la significatividad debe asociarse a estudios cualitativos. Por todo cllo, las investigaciones más interesantes están siendo aquéllas que exploran el problema desde una variedad de perspectivas, utilizando metodologías distintas tanto en la recogida como en el análísis de los datos. 
En cuanto a la estructuración del artículo para su publicación, conviene tener en cuenta ciertas pautas que sin duda facilitan la comunicación. Entre ellas destacaríamos la necesidad de referirse a:

- El marco teórico. Habitualmente se generaliza y se simplifica demasiado. Acostumbra a haber poca reelaboración de los referentes y no se justifican suficientemente en relación con el estudio concreto. Tampoco se acostumbra a distinguir entre el modelo de ciencia, el modelo de aprendizaje y el modelo de enseñanza y no se explícita el propio punto de vista. Por fin, se tiende a citar siempre a los mismos autores y a los mismos artículos.

- Los problemas o preguntas planteados. Es necesario justificar su significatividad y valorar las semejanzas y diferencias con otros trabajos que posiblemente se hayan realizado en el mismo campo.

- La metodología. Debe ser rigurosa. Ello implica que cualquier afirmación pueda ser validada a partir de los datos y de los referentes expuestos. En especial, es importante profundizar en los sistemas de organización de los datos. Los mecanismos utilizados para categorizarlos deberían estar bien justificados distinguiendo, siempre que sea posible, entre diferentes posibilidades. También deberían evitarse clasificaciones que no explican, por ejemplo, la distinción entre datos que se consideran «correctos» y los «alternativos». El problema de la investigación didáctica no es tanto verificar su existencia como llegar a comprender por qué se dan unos y otros y cuáles son sus características.

- Las conclusiones. Es importante distinguir entre resumir los resultados de Ia investigación e interpretarlos. Muchas veces, esta interpretación se debe plantear más como hipótesis de trabajo, que puede guiar futuras investigaciones, que como afirmaciones no siempre demostrables con los datos expuestos. Las opiniones expresadas deben contrastarse con los resultados de la investigación, con otros trabajos antecedentes y con los referentes teóricos.

También creemos que es importante profundizar en la organización y redacción del texto. Muchos artículos, con temas interesantes, no pueden aceptarse porque no se entienden ya sea debido a que no están bien estructurados, ya sea porque las frases están mal construidas.

\section{PROCESO DE SELECCIÓN DE LOS ARTICULOS PUBLICADOS}

Cada año se reciben más de cien artículos de los que se pueden publicar unos treinta. Ello indica que el proceso de selección es complejo y no siempre fácil. De él se responsabiliza el consejo de redacción y, en concreto, cada uno de sus miembros toma las decisiones en relación con los artículos que le corresponden en función de su propio campo específico de investigación.
Cada artículo es revisado como mínimo por dos especialistas en la temática planteada, que emiten su opinión sin conocer cuáles son los autores.

Con el fin de orientar el análisis, los asesores y asesoras reciben una sugerencia de indicadores para orientar su revisión de originales a ser publicados en la revista Enseñanza de las Ciencias. Son los siguientes:

a) Relevancia del trabajo para la didáctica de las ciencias o de las matemáticas

- Significatividad del problema analizado.

- Contribuciones o reflexiones novedosas en algún campo.

- Originalidad de la investigación o de la reflexión.

- Calidad de la revisión bibliográfica en relación con la temática tratada.

(Si el tema del artículo no es relevante, difícilmente podrá ser publicado.)

\section{b) Marco teórico y revisión bibliográfica}

- Suficiencia en la justificación del marco teórico.

- Especificidad de la bibliografía citada (por contrapo. sición a referencias muy generales).

- Adecuación de la definición del problema y de su relación con trabajos anteriores.

- Pertinencia y complitud de la bibliografía citada ( $\sin$ citas superfluas).

- Corrección en el redactado de las citas bibliográficas (en el texto y al final).

c) Metodologia y resultados

- Coherencia entre el diseño y el problema estudiado.

- Adecuación de la muestra. Especificación de los criterios de su selección y de sus características.

- Adecuación de las técnicas para la toma de datos. Especificación de las mismas.

- Adecuación del método de análisis de los datos al problema.

- Comprensibilidad en la comunicación de los datos (uso de tablas, ilustraciones, figuras ...).

\section{d) Interpretaciones y conclusiones}

- Rigor, pertinencia y originalidad en el análisis de los datos.

- Adecuación de la interpretación y discusión de los datos, tanto en relación con los que confirman la tesis del trabajo como con los discrepantes. 
- Claridad y pertinencia en las relaciones entre las afirmaciones y los datos.

- Calidad de las conclusiones en relación con la enseñanza de las ciencias o de las matemáticas.

\section{e) Redacción}

- Adecuación del título al contenido del trabajo.

- Pertinencia del resumen.

- Coherencia entre los planteamientos explicitados en la introducción y en las conclusiones (entre estas dos partes del artículo se debería poder tener una buena visión de las aportaciones del trabajo).

- Claridad, comprensibilidad y corrección del texto redactado.

- Ajuste de la extensión a las normas de publicación de la revista y al tema planteado.

Se recomienda a los asesores y asesoras que sean exigentes en el análisis y valoración de los originales $y$, al mismo tiempo y siempre que sea posible, que proporcionen orientaciones para su mejora.

El miembro del consejo de redacción al que le ha correspondido emitir el informe final analiza los informes y los coteja con su propia opinión. Muchas veces, dicho informe se acompaña de algunas de las valoraciones recibidas, para facilitar ia mejora del artículo, si se da el caso, o para que los autores o autoras conozcan los motivos de la aceptación o del rechazo.

\section{SECCIONES DE LA REVISTA}

Los artículos recibidos se distribuirán en las siguientes secciones:

\section{- Investigación didáctica}

En esta sección se recogerán artículos fruto de investigaciones sustentadas en datos experimentales, de reflexiones teóricas fundamentadas que aportan nuevos puntos

\section{REFERENCIAS BIBLIOGRÁFICAS}

DONMOYFR, R. (1996). Educational Research in an Era of Paradigm Proliferation: What's a Journal Editor to Do?, Educational Researcher, 25(2), pp. 19-25.

DÖRFI.ER, W. (1993). Quality Criteria for Journals in the Field of Didactics of Mathematics. ESM, pp. 75-87. de vista o de revisiones sobre el estado de la cuestión en relación con alguna temática.

\section{- Innovaciones didácticas}

En esta sección se recogerán artículos que analicen experiencias concretas de aula, propongan actividades innovadoras surgidas a partir de determinados referentes teóricos (experimentales o de otro tipo) o planteen revisiones de contenidos científicos fundamentadas didácticamente.

\section{- Historia y epistemología de las ciencias}

En esta sección se incluirán, como hasta ahora, artículos que analicen aspectos relacionados con la historia o con la epistemología de las ciencias que tengan interés por sus relaciones con la didáctica.

\section{- Debates}

En esta sección se editarán artículos que, alrededor de un mismo tema, planteen puntos de vista diversos o críticas a artículos publicados en números anteriores.

\section{- Resúmenes bibliográficos}

En este apartado se recogerán escritos que resuman el contenido de un conjunto de artículos relacionados con una misma temática o similares.

- Además se continuará con la sección de Informaciones y noticias, en la que se recogerán resúmenes de tesis doctorales, anuncios de congresos, referencias de grupos de trabajo, libros publicados, etc.

\section{REFLEXIONES FINALES}

Somos conscientes de la dificultad de gestionar todo este conjunto de factores complejos; sin embargo, asumimos con ilusión el reto de impulsar la publicación de la revista Enseñanza de las Ciencias cada vez con más rigor, más exigencia fundamentada. Nos resultaría de gran ayuda conocer las opiniones de autores y lectores en torno a estos aspectos que pueden constituir un tema de debate.
DUSCHL, R.A. (1994) Editorial Policy Statement and Introduction, Science Education, 78(3), pp. 203-208.

GILBERT, J.K. (1994). On the significance of journals in science education: the case of IESE, Int. J. Sci. Educ, 16(4), pp. 375-384. 
GIMENO SACRISTÁN, J. (1983). Planificación de Ia investigación educativa y su impacto en la realidad, en La enseñanza: entre la teoría y la práctica. Madrid: Akal.

KILPATRIC, J. (1981). Research on mathematical learning and thinking in the United States. Reserches en Didactique des Mathématiques, 2(3), pp. 363-379.
LESTER, F.K. (1993). Envolving criteria for judgind the quality of research reports in mathematicseducation. Escrito preparado para la conferencia "What is Research and What Are Its Results?», University of Maryland.

SMITH, M.U., WANDERSEE, J.H. y CUMMINS, C.L. (1993). Comments and criticism. Journal of Research in Science Education Teaching, 30(2), pp. 209-211. 
. 